\title{
LIGHTWEIGHT AND ENERGY-AWARE WIRELESS MESH ROUTING FOR AUTONOMIC INTERNET OF THINGS NETWORK
}

\author{
Lukman Rosyidi, Muhamad Asvial and Riri Fitri Sari \\ Department of Electrical Engineering \\ Faculty of Engineering, Universitas Indonesia \\ Kampus UI, Depok 16424, Indonesia \\ lukman.rosyidi@ui.ac.id, asvial@eng.ui.ac.id,riri@ui.ac.id
}

\begin{abstract}
This paper presents our proposed routing mechanism for wireless mesh sensor network which support autonomic Internet of Things (IoT). Autonomic IoT is the creation of self-management capability in the IoT system by embedding some autonomic properties, with the goal of freeing human from all details of the operation and management of the system while providing the system to always operate properly. The proposed routing provides a mechanism to embed some autonomic properties in the IoT edge network, i.e. self-configuration, self-healing, selforganization, and self-energy-awareness. It supports the IoT system to face the challenge of resource constraint of IoT nodes, as it becomes one of the top challenges in IoT edge network. A hierarchical routing fashion with residual energy consideration is used to form a lightweight routing algorithm. We provide a validation through computer simulations to investigate the routing performance for large number of IoT sensor nodes. We take an energy-aware straight path routing as the comparison. As the result, our proposed routing successfully produces longer network lifetime and lower total energy consumed.
\end{abstract}

Keywords - Internet of Things, autonomic computing, routing protocol, selfhealing, self-organization, self-energy-awareness

\section{INTRODUCTION}

Internet of Things (IoT) is a global network of various physical devices which are connected to the internet and then do the collection, exchange and processing of data. The IoT envisions a complex system whose purpose is to interconnect sensors and smart devices in such a way that makes them intelligent, programmable and more capable interacting with humans by providing useful services [1].

An IoT system usually consists of a large number of sensor nodes, one or more gateways, and a server or cloud platform in the Internet. The sensor nodes and the gateway are commonly referred as the IoT edge network. The sensor nodes capture the information from the surrounding environment, whereas the gateway is the point of sink that collects the data before it is sent over the Internet to the server or the cloud platform of IoT.

Some major challenges for IoT system are related to the network infrastructure of IoT edge network. The number of IoT nodes that can join the network can reach a very large number, which should be anticipated by the network mechanism. The IoT network can be heterogeneous. It is possible to have diversity in the network architecture and the protocol used by the nodes. The decision of network topology and protocol used may differ from

Received: December 15, 2018

Reviewed: February 5, 2019

Accepted: February 10, 2019

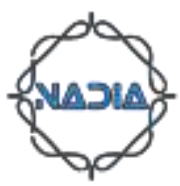


one case to other, to adapt to the condition and the needs of communication. An IoT system consists of many resource-constrained nodes that have limited energy, processing power, and memory. Some types of IoT device may also have mobility, which will follow an object's position. The IoT network is also vulnerable to malicious attack, so that the safety and the quality of information are important. It will be related to some aspects of security, privacy and trust. These challenges urge more research and development in IoT.

Energy constraint is one of the top issues in IoT edge network. The operation of IoT nodes, including the data communication, should support long life and equal energy residual among nodes in the network. This is because there is a high possibility of missing information from any sensor node that die because run out of energy at any time. The network lifetime measurement becomes important as it defines how long the IoT edge network can survive before one of its sensor node die. In most implementation, it is preferred to have the sensor nodes to die at almost the same time, because it means longer network lifetime. Furthermore, the replacement of batteries of all nodes can be done at the same time.

Recent research and development of wireless communication seek for answer for the energy constraint challenge faced by the IoT edge network through the design of the communication protocol and algorithm. Various protocol designs were proposed to establish an intelligent system [2]-[7]. Some of them are inspired by natural and biological systems, that can make the system to self-manage the complexity based on the objectives and the rules set by human. These protocols embedded the autonomic property into the system for various tasks and activities, such as network adaptation, network organization, energy management, network optimization and network protection.

This paper presents a proposed routing mechanism for network communication protocol of IoT edge network which can support the autonomic IoT. We organized this paper into five sections. Section 1 gives the introduction to the topic. Section 2 explains the related work. Section 3 explains the characteristics of an IoT mesh network. Section 4 explains the proposed protocol design to support the autonomic IoT mesh network. Section 5 discusses simulation result. Section 6 provides the conclusion and the future work.

\section{RELATED WORK}

Many studies have been conducted to propose an optimum routing protocol for wireless mesh network. Each protocol has its own algorithm. Some algorithms are based on organized works that are detailed documented and formalized as protocol standards, while some others are pragmatic approaches developed by community and publicly available.

Some of the developed protocols for wireless mesh network are adapted from protocols that are designed for ad hoc networks, such as Dynamic Source Routing (DSR) [8], Optimized Link State Routing (OLSR) [9], and Ad-hoc On-demand Distance Vector routing (AODV) [10]. OLSR is proactive which maintain fresh list of routes in the network, while DSR and AODV are reactive which find a route on demand by flooding the network with Route Request packets.

Better Approach To Mobile Ad-hoc Networking (BATMAN) [11] is an example of proactive routing protocol for wireless mesh network. It uses a distance-vector approach and a routing metric, which incorporates the reliability of the radio links. In later development, BATMAN-adv was introduced for routing on layer 2. BATMAN and BATMAN-adv have received attention in the scientific community. Works in [12] showed that BATMAN outperforms AODV.

Hybrid Wireless Mesh Network (HWMN) protocol, which is based on 802.11s standard, combines reactive and proactive routing for wireless mesh network. It relies on a peer link management protocol by which each mesh point discovers and tracks neighboring nodes. Work in [13] showed that HWMN had better packet delivery ratio than BATMAN-adv, but BATMAN-adv can give better delay and more throughput. 
Despite the availability of scalable routing protocols, the need for energy-aware routing protocol for wireless mesh network has increased, especially in widespread applications of Wireless Sensor Network and IoT. The Physarum-based Routing Scheme (P-bRS) [14] is one of the protocols. Our work has focus on developing a better energy-aware routing protocol for the implementation in IoT edge mesh network.

\section{ROUTING IN AN IOT MESH NETWORK}

An IoT system usually consists of a large number of sensor nodes, one or more gateways, and a server or cloud platform in the Internet. The sensor nodes and the gateway are commonly referred as the IoT edge network.

The IoT gateway is the point of sink that collects data before it is sent over the Internet to the server or the cloud platform of IoT. It is usually in the form of device that has large computing and energy resource. The device can be a computer with a high processing power and high memory capacity with sustainable supply of energy because it is connected directly to a power source. It provides the required communication interface for delivery to the Internet, either via Wi-Fi, Ethernet or cellular communication.

The IoT nodes usually are devices that have limited energy resource, processing power, and memory. The device can be a microcontroller equipped with sensors and a wireless communication module, with the energy supply from a battery. It may also be equipped with an electronic module that can harvest energy from the environment.

In a network, the constructed network topology can be a tree, star, or mesh. A large network commonly uses the mesh network topology. This topology has an advantage in term of connectivity and reliability because any sensor node can be used as a relay point for the data before it is sent to the gateway. A data transmission from distant locations can be done through a multi-hop communication.

Wireless mesh IoT edge network has a similar network topology with the ordinary wireless mesh network, but there are some differences because of the nature of constrained nodes in IoT edge network, as shown in Table I. The constrained node is a node where some of its features and capability are limited, often due to cost constraints or physical constraints on characteristics such as size, weight, available power and energy. The tight limits on power, memory, and processing resources lead to hard upper bounds on state, code space, and processing cycles, making optimization of energy and network bandwidth usage a dominating consideration in all design requirements [15],[16].

Table I. Differences Between Ordinary Mesh Network and IoT Mesh Network

\begin{tabular}{|l|l|l|}
\hline $\begin{array}{l}\text { Network } \\
\text { characteristics }\end{array}$ & $\begin{array}{l}\text { Ordinary wireless } \\
\text { mesh network }\end{array}$ & $\begin{array}{c}\text { Wireless mesh IoT } \\
\text { edge network }\end{array}$ \\
\hline Direction & $\begin{array}{l}\text { From and to any node } \\
\text { in the network }\end{array}$ & $\begin{array}{l}\text { Mostly from sensor } \\
\text { nodes to gateway }\end{array}$ \\
\hline Traffic & $\begin{array}{l}\text { Can be any type of } \\
\text { traffic }\end{array}$ & $\begin{array}{l}\text { Low rate and } \\
\text { intermittent traffic of } \\
\text { sensor data }\end{array}$ \\
\hline $\begin{array}{l}\text { Main design } \\
\text { objective }\end{array}$ & $\begin{array}{l}\text { High reliability } \\
\text { network }\end{array}$ & Low power network \\
\hline $\begin{array}{l}\text { Main } \\
\text { performance } \\
\text { measurement }\end{array}$ & $\begin{array}{l}\text { Packet loss, delay, } \\
\text { throughput }\end{array}$ & $\begin{array}{l}\text { Network lifetime, } \\
\text { energy consumption }\end{array}$ \\
\hline
\end{tabular}

A common mesh network can have traffic direction from and to any node in the network and carry any type of data. The main network performance measurements usually are packet loss, delay and throughput. Meanwhile, the traffic direction of IoT edge network is 
from the sensor nodes to the gateway, carrying measurement data of sensor which is small, low rate, and intermittent. Most IoT edge networks are designed for low power network. Therefore, the network lifetime becomes an important network performance measurement.

\section{LIGHTWEIGHT AND ENERGY-AWARE WIRELESS MESH ROUTING}

We propose Lightweight Energy-Aware Routing (LEAR), which is specifically designed for Internet of Things edge network. It has some unique characteristics that are different with common wireless mesh network. One major concern in IoT is the energy constraint of the nodes. The node's operation should have low energy consumption, including the operation for communication. A data transmission from distant locations can be done through a multi-hop communication. However, there is a possibility of data missing on communication at any time because the intermediate node may die or change its position.

The main idea of LEAR is improvement on the next-hop selection algorithm that must consider residual energy of neighboring nodes in addition to the most efficient path to destination. The algorithm of the selection should be simple and lightweight, such that it can be implemented in any low power device nodes without making a long processing time or consuming much energy for the computational efforts.

\subsection{NETWORK MODEL AND PROBLEM FORMULATION}

Our network model is an IoT network which consists of $n$ nodes as end device and capable to become relay node for data transmission. In graph, it is modelled as $G=(V, E)$, where $V$ is set of nodes as vertices and $E$ represents a set of links between node pairs. The message is sent from a source $s \in V$ to a destination $d \in V$ which is the IoT gateway, by following these rules:

1) For each node $i \in V$ in the network, there exist $B$ as a set of one-hop neighbors for the sender node $i$ that has sufficient residual energy.

2) The message can be transmitted along one link $(s, d) \in E$ if $d$ is an element of $B$. Otherwise, it should be transmitted along a multi-hop route made up of a link $(s, i)$ and a set of route $[i, d]$, where $i$ is a node in $B$ and $(s, i)$ is a link in $E$.

3) The route set $[i, d]$ represents the routes from node $i$ to node $d$ through a subnet $S=(V-\{s\}, E-\{(s, i): i \in B\})$.

4) The route finding in subnet $S$ is also following the rule 1 to 3 above recursively.

Each of the node $i$ in $V$ has energy constraint, so that its residual energy (RE) of each nodes is limited by $0 \leq \mathrm{RE} \leq 1$, except for the gateway where $\mathrm{RE}$ is always set to 1 because it always connected to the source of energy. The objective of the routing is to deliver as many messages from IoT nodes to the IoT gateway before any of the nodes in network becomes run out of energy. Therefore, the strategy of the path selection becomes important to make energy equilibrium among the network nodes.

\subsection{PACKET DATA FORMAT}

The packet data format for the proposed routing protocol is shown in Figure 1. Each packet header for multihop routing will contain the information of Network ID (NI), Nexthop Address (NA), Source Address (SA), Originator Address (OA), Packet Type (PT), and Data Length (DL). This header is followed by the payload data and a Cyclic Redundancy Check (CRC) code. 


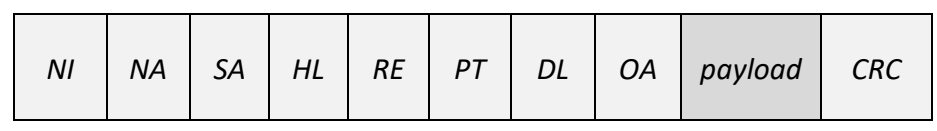

Fig. 1 Packet Data Format

The self-energy-awareness is embedded to the routing decision by adding energyrelated information to the packet header, i.e., Residual Energy (RE). This information together with Hierarchical Level (HL) will be used in determining the next-hop address. By considering this RE information, the routing mechanism will support the self-energy-awareness of the network.

\subsection{ENERGY AWARENESS IN PATH SELECTION}

Some studies have introduced routing algorithms that consider the residual energy of the neighboring nodes in the path selection. One of them, which is lightweight, is Physarumbase Routing Scheme (P-bRS). P-bRS is inspired by flux equation of Physarum model [14]. In addition to residual energy, P-bRS use the angle of deviation to destination as the parameter to assist to the right direction and the most efficient path to destination.

Equation (1) shows the P-bRS formulation for scoring the neighbor nodes as written in [14].

$$
Q_{i j}=\frac{D_{i j} \cdot \Delta P}{L_{i j}}=\frac{k \cdot \mathrm{ER}_{j}+(1-k) \cos \theta_{j i d}}{L_{i j}} .
$$

$Q_{i j}$ is the flux of communication through the wireless link and $E R_{j}$ is the value of energy residue of the relay node, while $L_{i j}{ }^{\alpha}$ is the signal attenuation for the distance from the sending node to the relay node. $\theta_{j i d}$ is the angle of deviation of the relay node to the destination. The constant $k$ is a weight factor, which is used to adjust the proportion of $E R_{j}$ and $\cos \theta_{j i d}$. The neighbor with the largest $Q_{i j}$ will be selected as the next hop.

The Equation (1) is not easy to implement because each node has to know the neighbor's coordinates and calculate $\theta_{j i d}$. Moreover, the use the angle of deviation, which prefers straight path to destination, can mislead to inefficient route, if there is an absence of relay node in the straight path.

In LEAR, we propose to use information of Hierarchy level (HL) in determining the next-hop address. The data packet will prefer to flow from a higher level node to a lower level node. This parameter is combined with Residual Energy (RE) parameter, so that the routing should prefer to route via nodes that have higher residual energy rather than those that have lower residual energy.

We propose that there is a central node that initiates network construction process to form the network tree. This role can be assigned to the IoT gateway, since it becomes the destination for all IoT device nodes. The Hierarchical Level (HL) begins from the gateway to all IoT nodes, start from the nearest ones that have fewest hop to the further ones that need more hops from the gateway.

This HL value is identical with the minimum number of required hops to send message from a node to the IoT gateway as the destination. Figure 2 shows the example value of HL for the nodes in a network. This HL number is determined on network initialization or re-initialization process, starting from the IoT gateway node $(\mathrm{HL}=0)$ and incremented for each next hop. By using this HL assigning, the routing mechanism will support the selforganization of the network. 


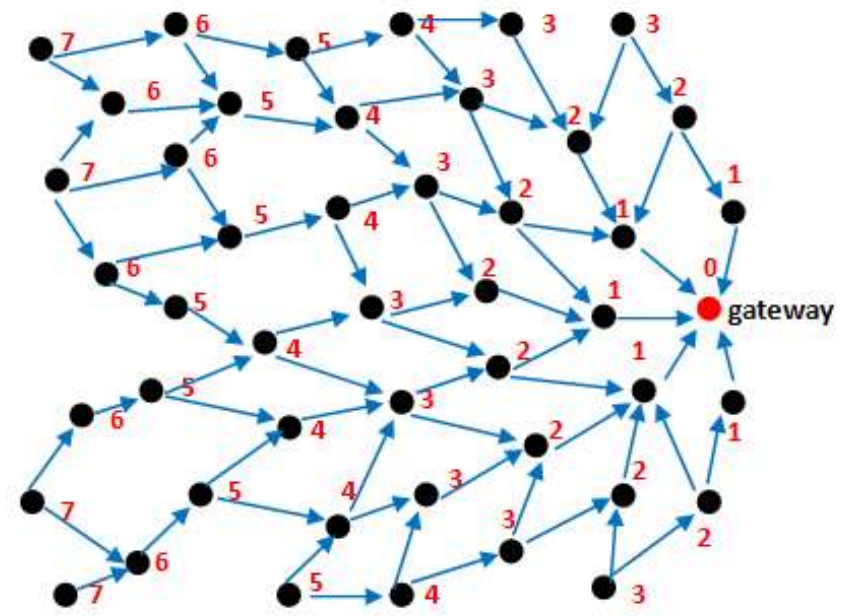

Fig. 2 Hierarchical Level of Nodes

IoT nodes usually are battery-powered device. The Residual Energy (RE) is required for LEAR algorithm and each node can get the information from the electronic of the device. Since the RE will be vary between different hardware device along with different type of battery used, we take the normalized value of RE to be used in our path selection algorithm.

$\widehat{R E}_{i}=\frac{\text { current battery level of node } i}{\text { max battery level of node } i}$

For every sender node $s$, it needs to calculate the score of $Q_{s i}$ for every neighbors node $i \in B$. The next-hop selection procedure is simplified for lightweight computation and expressed in the following equations.

$$
\begin{aligned}
& Q_{s i}=a \times\left(H L_{s}-H L_{i}\right)+(1-a) \times \widehat{R E}_{i} \\
& i_{\text {selected }}=\arg \max _{i \in B}\left(Q_{s i}\right)
\end{aligned}
$$

The score $Q_{s i}$ is a weighted-average value between the differences in HL and normalized RE. Then, the elected next-hop is node $i$ where its $Q_{s i}$ is maximum among other $Q_{s i}$ in $B$. Figure 3 shows a possible result of this path selection process.

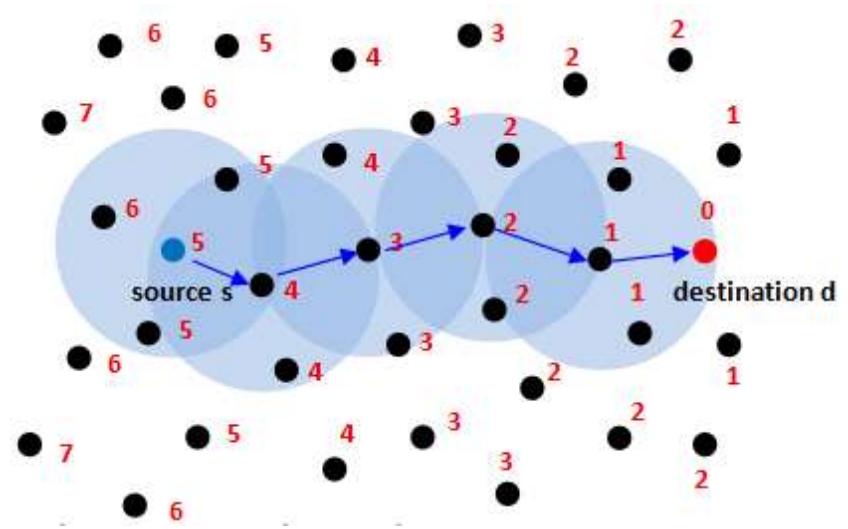

Fig. 3 Example Result of LEAR Path Selection 


\subsection{THE ROUTING ALGORITHM}

For LEAR implementation, there are four states of communication activity in the node i.e., initialization, receive, transmit, and idle. In the first time, a new node needs to execute network initialization process to join the network. If it receives a packet from any of its neighbors, it will update the sender information (HL and RE) in its memory and then examine the target address (NA) field in the packet. If the NA matches with its ID, it then executes routing and transmission process. The routing determines the next hop address (NA) field and do the transmission if NA can be determined. Otherwise, if NA cannot be determined then the network re-initialization process should be triggered and data should be buffered and scheduled for next transmission. In a certain periodic of time, the node will also automatically trigger the network re-initialization to update its connection to the network.

Figure 4 shows the state chart diagram for the network initialization process. A node that is not connected to the network or lost its connection should broadcast the information request packet. This request is similar to hello message in other protocols but adds the information of $\mathrm{HL}$ and RE to be known and stored by its neighbors for use of routing process. In a certain period of time, the node will have the HL information of all neighbors. It will then assign HL for itself by taking an increment on the lowest HL in the HL table of neighbors, which means it will become the child of the nearest HL neighbor to the gateway in the current connection. By doing this mechanism, it will support the self-configuring and self-organization of the network.

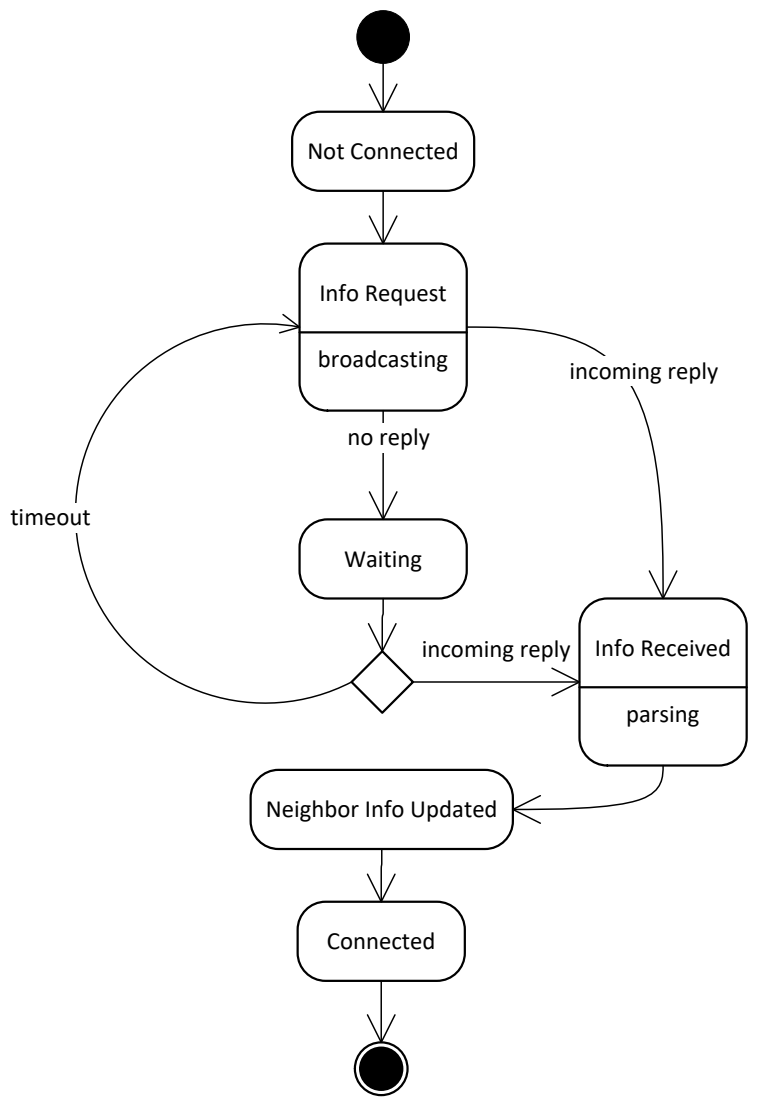

Fig. 4 State Chart Diagram for Network Initialization Process

The information request packet has a unique packet type (PT) code that the neighbors need to reply by sending similar information. If no reply received in a timeout period then the node should resend this information request broadcast. Any reply from any neighbors 
should be parsed to update the neighbor information table. The reply should also be received by any other neighbors that will do the update of information of their neighbor information tables. Each node also can renew its HL value based on the updated information. By doing this mechanism, the neighbor table in every node in the network will always be kept updated by the execution of network initialization process from any neighboring node, thus it supports self-healing and self-organization of the network.

Figure 5 shows the state chart diagram for receiving process. An incoming packet data will be buffered and parsed. All nodes can get the information of NI, NA, SA, HL, RE and PT. Furthermore, every node should update its neighbor information table based on information of SA, HL and RE in each incoming packet received if it has the same network ID (NI). It then checks whether its ID matches NA, which means the packet is sent for the node. Otherwise, the packet is not for the node and should be cleared from its buffer. If the packet is addressed for the node, then ACK is sent and state will continue to routing and transmission process. The exception is only for the gateway where $\mathrm{HL}=0$, in which the data has been received in the final destination and the routing process is complete. The gateway can get the Data Length (DL) and the Originator Address (OA) as well as the payload data and its CRC.

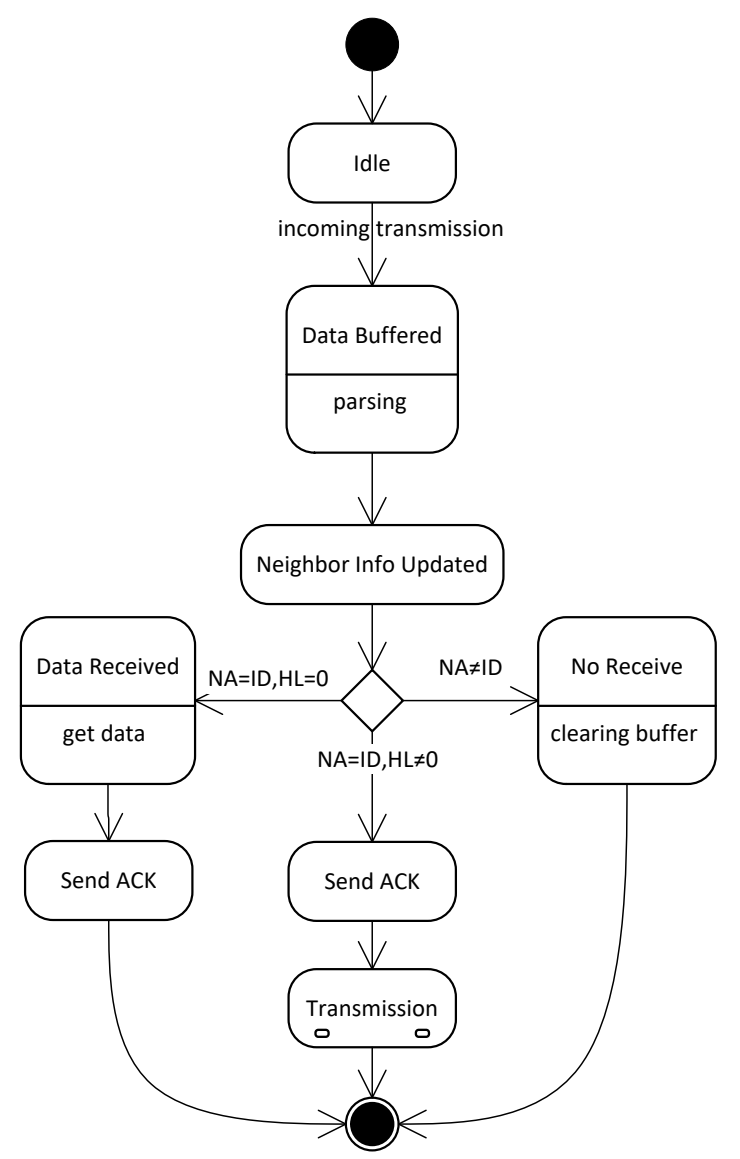

Fig. 5 State Chart Diagram for Receiving Process

Figure 6 shows the state chart diagram for transmission process. This process is for the sending data packet or routing data packet from the neighbor. The node need to execute routing calculation based on the information in its neighbor information table using Equation (2)-(4) to get the NA for the transmission. If the NA does not exist because no neighbors found in the last initialization process, it should trigger network re-initialization process. Otherwise, it can continue to send the packet with the obtained $N A$ along with updating $S A$, HL and RE with its own information. A 
short ACK packet from the receiver is required in order to confirm the acceptance of the packet. If no ACK received in a certain period of time, which means the neighbor may have disappeared or run out of energy, it should trigger the network reinitialization process and reschedule the transmission. By doing this mechanism, it will support the self-healing and self-organization of the network.

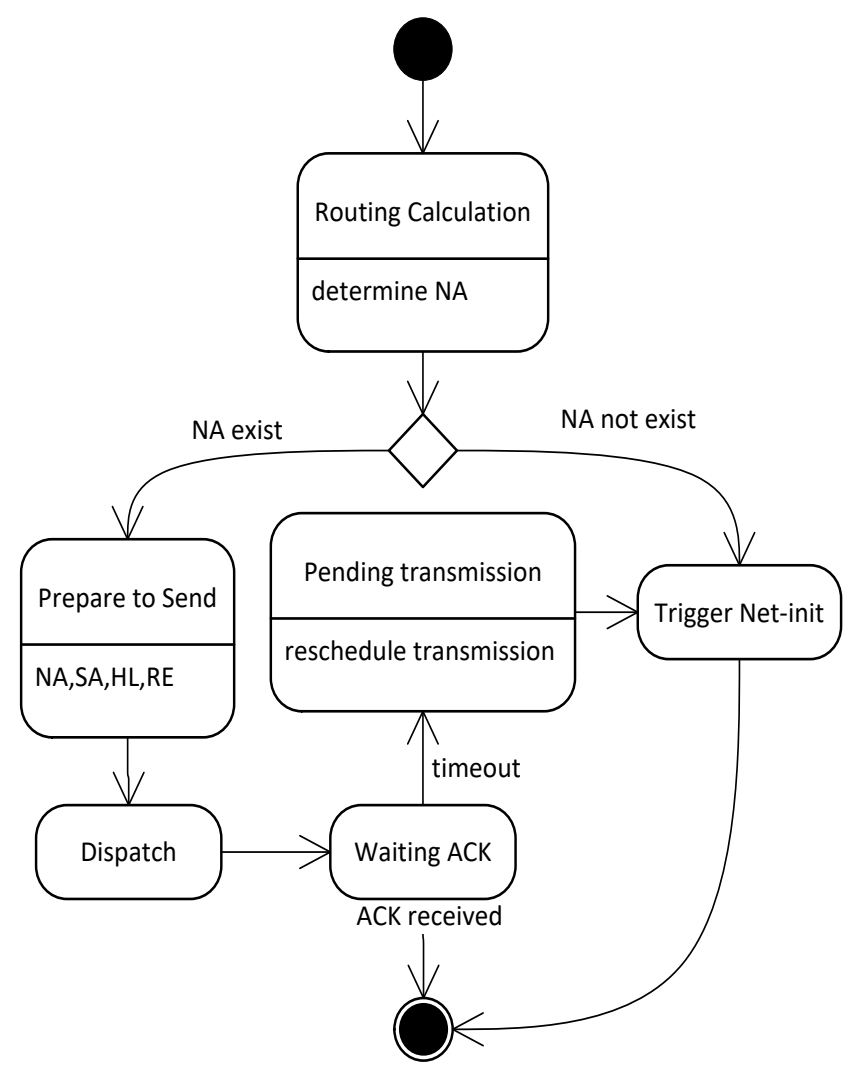

Fig. 6 State Chart for Transmission Process

\section{SIMULATION RESULTS}

We use computer simulation to validate the performance of LEAR in a large number of nodes using Matlab software tools. The objective of the simulation is to evaluate the routing performance of LEAR and compare the result with other energy-aware wireless mesh routing for IoT edge network, especially in term of network lifetime. For this purpose, we take Physarum-based Routing Scheme (P-bRS) as the comparison. P-bRS uses an energy-aware straight path routing approach [14].

The network lifetime is an important measurement for IoT network. It is the measurement of how long all nodes in a network is fully operational before at least one of the nodes dies because it run out of energy. Every node is considered to be important for the network and contribute valuable information. The routing protocol should support to maintain the energy equilibrium of the network, to achieve the longest network lifetime.

For the simulation purpose, we use 380 nodes which are deployed in $2000 \times 2000 \mathrm{~m}^{2}$ in a uniform distribution. Our protocol design does not include the physical and datalink layer, therefore the link quality is assumed to be the same for all nodes. Table II shows our simulation parameters. 
Table I. Simulation Parameters

\begin{tabular}{|l|l|}
\hline Parameter & Value \\
\hline Simulation area & $2000 \times 2000 \mathrm{~m}^{2}$ \\
Physical layer & 802.11 \\
Distribution of nodes & Uniform \\
Number of nodes $(N)$ & 380 \\
Transmitter range & $120 \mathrm{~m}$ \\
Transmitter power & $0.0035 \mathrm{~W}$ \\
Antenna & Omnidirectional \\
Weight factor $(a, k)$ & $a=0.1, k=0.9$ \\
Initial energy $\left(E R_{0}, R E_{0}\right)$ & $1 \mathrm{~J}$ \\
Path loss exponent $(\alpha)$ & 2 \\
\hline
\end{tabular}

Figure 7 shows the network lifetime result for $a=0.1$. This value of $a$ is selected as means of high consideration of residual energy of the next hop address. All nodes in the network are alive until some nodes that frequently used in routing start to run out of energy. For the P-bRS, the first node dies in round of 279, while in LEAR this happens in round of 341. Hence, the network lifetime in LEAR is $22 \%$ longer than that in P-bRS.

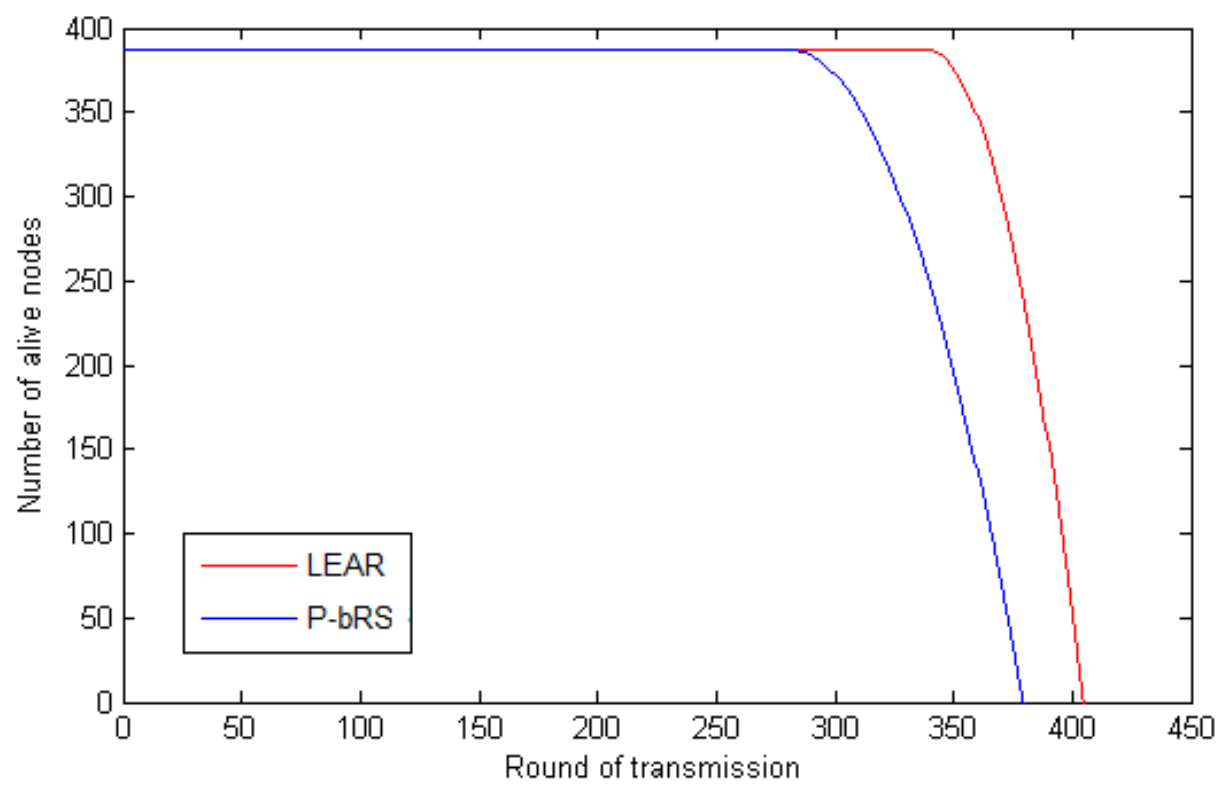

Fig. 7 Simulation Result for Network Lifetime

Figure 8 shows the total accumulated energy consumption over time for P-bRS and LEAR where $a=0.1$. It shows that the LEAR is more energy efficient than P-bRS. For the P-bRS, the total energy consumed at round of 348 is $100 \mathrm{~J}$, while for LEAR is $92 \mathrm{~J}$. Hence, the LEAR is $8 \%$ more energy efficient than P-bRS. The main factors that influence the energy consumption are the required power for executing the routing mechanism in the nodes and the number of hop in data delivery. 


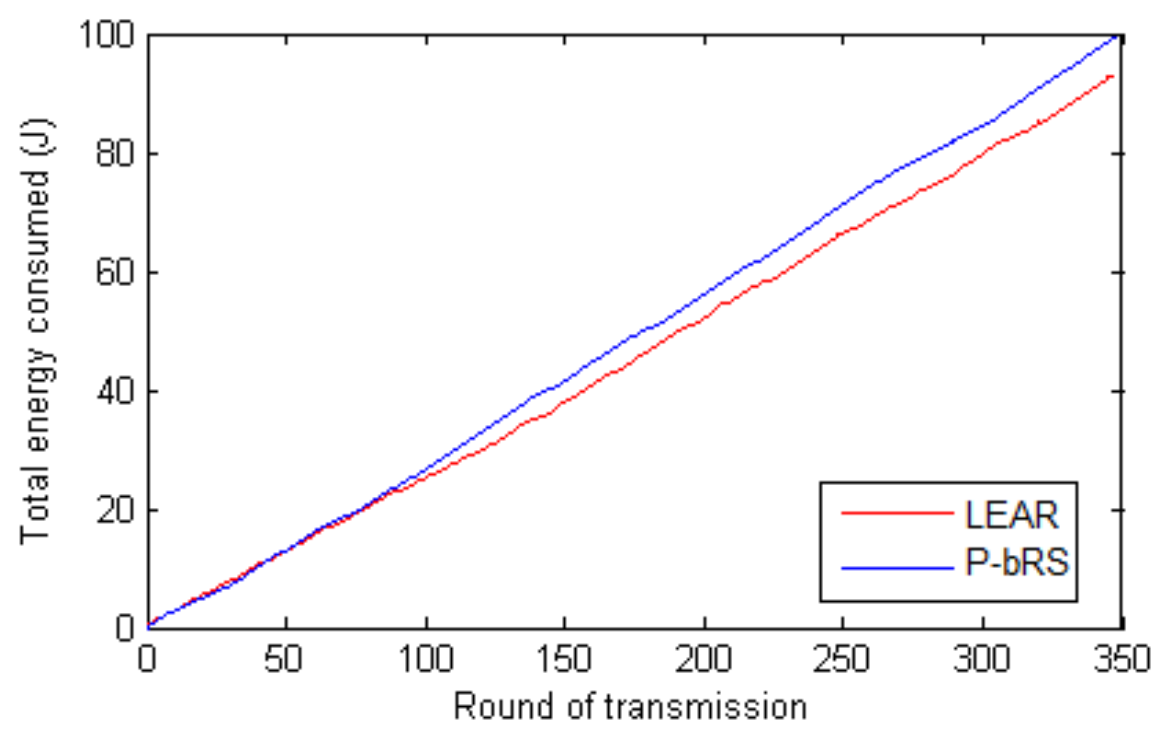

Fig. 8 Simulation Result for Total Energy Consumed

The difference in the results of network lifetime and total energy consumption is caused by the difference of the path selections in routing between LEAR and P-bRS. The data transmissions make some nodes near the gateway become exhausted for being use as relay node in routing. P-bRS directs the route to the lowest angle of deviation to destination. The neighbor nodes will have different score because of the difference in position. The nodes that are positioned in the straight path to destination will have better score and higher possibility to become the next hop, as they will also run out of energy faster. LEAR directs the route to the positive difference in hierarchical level. The neighbor nodes in the same hierarchical level will have the same possibility to be selected as the next hop. Therefore, the selection will rely more on the residual energy of the nodes. This make the use of LEAR can produce better energy equilibrium among the nodes.

\section{CONCLUSIONS}

We have presented the Lightweight and Energy-Aware Routing (LEAR) as our proposed routing mechanism for IoT wireless mesh network. The mechanism aims to provide longer network lifetime and lower energy consumption. Instead of using a straight path to destination approach like P-bRS, LEAR uses a hierarchical routing approach. It is also simplified to provide easier implementation and a lightweight computation for low power IoT device nodes.

The simulation results validate that LEAR successfully produces better network lifetime and energy efficiency compared to P-bRS. LEAR successfully improves up to $22 \%$ of network lifetime and $8 \%$ of total energy consumption compared to P-bRS.

For the future work, we will validate LEAR in real testbed to investigate some important aspects of its mechanism in the real implementation. This is important to assure that the protocol design meets the requirements for routing in IoT low power network.

\section{ACKNOWLEDGMENTS}

The research reported in this paper was supported by Ministry of Research, Technology and Higher Education of the Republic of Indonesia, under PTUPT Grant No. 505/UN2.R3.1/HKP05.00/2018. 


\section{REFERENCES}

[1] R. Minerva, A. Biru and D. Rotondi, "Towards a Definition of the Internet of Things (IoT)", IEEE Internet Initiative, (2015)

[2] J. Habibi, A. Ghrayeb and A. G. Aghdam, "Energy-Efficient Cooperative Routing in Wireless Sensor Networks: A Mixed-Integer Optimization Framework and Explicit Solution," in IEEE Transactions on Communications, vol. 61, no. 8, (2013), pp. 3424-3437.

[3] K. N. Kannan and B. Paramasivan, "Development of energy-efficient routing protocol in wireless sensor networks using optimal gradient routing with on demand neighborhood information," International Journal of Distributed Sensor Networks, (2014).

[4] D. R. Cañas, A. L. S. Orozco, L. J. G. Villalba, and P. Hong, "Hybrid ACO Routing Protocol for Mobile Ad Hoc Networks," International Journal of Distributed Sensor Networks, (2013).

[5] M. Xu and G. Liu, "A multi-population firefly algorithm for correlated data routing in underwater wireless sensor networks," International Journal of Distributed Sensor Networks, (2013).

[6] E. Amiri, H. Keshavarz, M. Alizadeh, M. Zamani, T. Khodadadi, "Energy Efficient Routing in Wireless Sensor Networks based on Fuzzy Ant Colony Optimization," International Journal of Distributed Sensor Networks, (2014).

[7] H. Simaremare, A. Abouaissa, R. F. Sari, and P. Lorenz. "Performance analysis of optimized Trust AODV using ant Algorithm," in IEEE International Conference on Communications (ICC), pp. 18431848. IEEE, (2014).

[8] D.B. Johnson and D.A. Maltz, "Dynamic source routing in ad hoc wireless networks," Mobile Computing, vol. 353, (1996), pp. 153-181.

[9] P. Jacquet, P. Muhlethaler, T. Clausen, A. Laouiti, A. Qayyum, and L. Viennot, "Optimized link state routing protocol for ad hoc networks," Multi Topic Conference IEEE INMIC 2001. Technology for the 21st Century. Proc. IEEE International, (2001), pp. 62-68.

[10] C.E. Perkins and E.M. Royer, "Ad-hoc on-demand distance vector routing," Proceedings of the 2nd IEEE Workshop on Mobile Computing Systems and Applications, vol. 2, (1999), pp. 90-100.

[11] D. Johnson, C. Aichele, and N. Ntlatlapa, "A simple pragmatic approach to mesh routing using BATMAN,” 2nd IFIP Int. Symp. Wirel. Commun. Inf. Technol. Dev. Ctries., (2008), pp. 10.

[12] D. Seither, A. König, and M. Hollick, "Routing performance of wireless mesh networks: A practical evaluation of BATMAN advanced," Proceedings of Conference Local Computer Networks, LCN, (2011), pp. 897-904

[13] M. S. Singh and V. Talasila, "A practical evaluation for routing performance of BATMANADV and HWMN in a Wireless Mesh Network test-bed," 2015 Int. Conf. Smart Sensors Syst. IC-SSS, (2015).

[14] M. Zhang, W. Wei, R. Zheng, Q. Wu. "P-bRS: a physarum-based routing scheme for wireless sensor networks", The Scientific World Journal, (2014).

[15] C. Bormann and M. Ersue and A. Keranen, "Terminology for Constrained-Node Networks", in Internet Requests for Comments (RFC) 7228, IETF, (2014).

[16] Pallavi Sethi and Smruti R. Sarangi, "Internet of Things: Architectures, Protocols, and Applications", Journal of Electrical and Computer Engineering, vol. 2017, Article ID 9324035, (2017). 